\title{
Stimulus package
}

\begin{abstract}
Therapies for Alzheimer's disease and other neurodegenerative diseases are desperately needed. Yet, a string of disappointments in the neurodegenerative therapy space has meant that several companies over the years have ended their investment in the field. Some companies have diversified their research and development (R\&D) models to hedge their bets. Maintaining this diversity to bring down the silos between big pharma and smaller research teams may be necessary to jumpstart and sustain progress in combatting neurodegenerative conditions.
\end{abstract}

$\mathrm{n}$ January this year, pharmaceutical giant Pfizer announced that it would be shuttering much of its neuroscience program, including R\&D into Alzheimer's disease and Parkinson's disease. The news was met with mixed reactions. Some have chastised the company for throwing in the towel at a crucial time when few effective therapies exist for neurodegenerative diseases. The failure rate of clinical trials for Alzheimer's disease, according to a 2014 report, is at $99 \%$, and so some others have come to expect such exits in the risk-filled field of neurodegeneration (Alzheimers Res. Ther. 6, 37, 2014). But Pfizer's decision was not for a lack of trying: Pfizer sponsored nearly 100 trials evaluating various Alzheimer's disease therapies in the past 20 years, according to ClinicalTrials.gov.

For the more than 10 million people worldwide suffering from Parkinson's disease and more than 40 million people thought to be afflicted with Alzheimer's disease, news of a major pharmaceutical company halting its work on these areas may seem devastating. But in the letter announcing the company's plans, Pfizer also revealed that it would be setting up a venture fund to invest in smaller biotech companies that are "conducting promising neuroscience research, particularly in areas of unmet need," in both Alzheimer's disease and Parkinson's disease.

The stakes for finding an effective therapy for Alzheimer's disease are high. No new agent-either for symptoms or underlying causes of the disease-has been approved in 15 years, and a February report estimated that 15 million people in the United States alone could have Alzheimer's disease-caused mild cognitive impairment by 2060 (Alzheimers Dement. 14, 121-129, 2018). Beyond the dismal failure rate of trials, some estimate the total cost of developing one new disease-modifying drug at $\$ 5.7$ billion, and so companies like Pfizer may be smart to outsource the early-stage research to smaller companies that often have the flexibility to go after innovative targets (Ann. N.Y. Acad. Sci. 1313, 17-34, 2014). Although Pfizer hasn't yet shared more details on this fund or which companies it will be funding, it seems that at least some of the risks of early $\mathrm{R} \& \mathrm{D}$ will be undertaken by the external companies. For investors, the amount of money needed to fund smaller biotech companies to do basic target discovery research, and therefore the associated risk, is often less than what it takes for larger companies to keep robust research programs funded.

Such alternative approaches may be crucial to include in a company's $\mathrm{R} \& \mathrm{D}$ portfolio when considering 'risky' fields like neurodegeneration. And Pfizer isn't the only company to take an alternate approach, nor is partnering with smaller firms the only alternative method. Amgen and Novartis, for instance, have been collaborating on a beta-site amyloid precursor protein-cleaving enzyme 1 (BACE1) inhibitor program for Alzheimer's disease since 2015, most recently announcing an expansion of this collaboration along with the Banner Alzheimer's Institute. AstraZeneca and Eli Lilly have similarly partnered up to work on a BACE1 inhibitor as well as an anti-beta-amyloid drug.

Novel ways of doing basic R\&D also offer companies the opportunity to explore untested avenues. Many of the therapies in development have focused heavily on leading targets like beta-amyloid, for example, with little to show in terms of clinical successes. Smaller companies, however, are focused on emerging targets such as those that inhibit inflammatory pathways (like Annexon Biosciences) and modulate metabolism (Accera, for example).

Companies such as Johnson \& Johnson and Amgen have invested in smaller, external research teams and, in the case of the former, even set up independent lab spaces around the country in the form of JLabs. In December, Amgen announced that it was funding a team at Cambridge, Massachusetts-based LabCentral, a shared laboratory space for small teams of scientists, working on treatment for the neurodegenerative disease amyotrophic lateral sclerosis. More big pharma companies could consider a nimbler approach to funding research teams focused on neurodegeneration. Large companies could even set up a collaborative space specifically for research into dementia and related conditions to help accelerate the early, target-driven research of which the industry is in need. Other creative solutions include the creation of the Dementia Discovery Fund, a venture fund set up in 2015 to invest in companies that are developing therapies for dementia. Its most recent, and perhaps most high-profile, investor was Bill Gates, who poured $\$ 50$ million into the fund.

Changes on the regulatory side could also help the current dearth of neurodegenerative therapies. The Neurotechnology Industry Organization, a trade organization for biotech companies specifically in the neuroscience space, has previously called for incentives for pharmaceutical companies-like priority reviews—from the US Food and Drug Administration (FDA). In 2013, the FDA released a guidance document for companies developing drugs for early stage Alzheimer's disease, but specific incentives solely for neurodegenerative diseases are something that the agency could consider.

Any basic R\&D effort into neurodegenerative therapies is going to require a long-term investment. Whether that $R \& D$ investment continues to be made internally or externally, the choice that companies make may mean the difference between having only a few players in the field with only a few targets to play with and having a truly diverse and robust set of options with which to tackle neurodegeneration. 